\title{
Redistricting to Maximize the Preservation of Political Boundaries
}

\begin{abstract}
JOHN R. BIRGE
Department of Industrial and Operations Engineering, University of Michigan

Redefining legislative districts is a task undertaken by the states after each census in order to ensure equitable representation. Many criteria have been proposed as objectives in forming districts but specific definitions of an optimal plan have not been enforced. In attempting to eliminate political concerns from the effort, the Michigan Supreme Court defined criteria based on the preservation of county and municipality borders. $\Lambda$ quadratic programming formulation is given for this problem, and a heuristic solution procedure is proposed.
\end{abstract}

In Reynolds v. Sims (377 U.S. 533, 583 (1964)), the U.S. Supreme Court ruled that the states should define the boundaries of legislative districts according to decennial census data in order not to violate the "one person, one vote" rule. The state legislatures undertake this redistricting activity and generally develop politically partisan plans. Avoiding political considerations becomes very difficult because many objectives may be used to justify alternative plans.

The following criteria are usually considered in evaluating redistricting plans:

(1) population equality: the districts are selected so as not to deviate beyond some limit from a mean population;

(2) compactness: the districts have regular shapes, being nearly square or nearly circular;

(3) contiguity: the districts are constructed so that one may travel between any two points in a district without leaving the district;

(4) political boundary integrity: the districts should preserve political boundaries as much as possible.

Contiguity and some standard of population equality are given the heaviest weight in redistricting plans. Possible dilution of minority voting strength is also considered and can be challenged in court as unconstitutional or under the Voting Rights Act even when equipopulous districts have been constructed (Engstrom, 1976). 
On challenges of redistricting plans based on violation of equal population ("one person-one vote"), the Supreme Court has considered plans with less than a $10 \%$ (of the mean district population) deviation from smallest to largest population district as essentially having equal population districts. In cases where the deviation is greater than $10 \%$ the states should show some justification for the deviation (Guida, 1982). The court has allowed up to a $16.4 \%$ deviation given the states' need to maintain existing political unit boundaries (Mahan v. Howell, 410 U.S. 315 (1973)).

Many optimization methods for redistricting have been considered with the goal of creating as nearly equally populous districts as possible. Garfinkel and Nemhauser (1970) proposed an algorithm for minimizing population deviation by generating all feasible districts. In their procedures, they start with population units and combine them into districts that satisfy population equality, contiguity, and other criteria. The difficulty with their approach, however, is that the number of such feasible districts may be extremely large and may make the problem intractable.

Other methods include Forrest's (1965) heuristic method of repeatedly dividing regions into equipopulous districts until the desired number of districts has been achieved. Weaver and Hess (1963) used a warehouse location model to create districts by starting with a group of district centers, assigning regions to the centers while maintaining populations within bounds, and then moving the centers to the population centroids of the new districts. When no further improvement is made, the algorithm stops. Nagel (1965) considered several criteria in his switching algorithm for trading along the borders of existing districts in order to improve a weighted objective of any of the criteria of population equality, compactness, or other political goals. These methods are very sensitive to starting conditions and may not lead to nearly optimal solutions.

The advent of these and other methods was hoped to alleviate the problems inherent in partisan choices of districts. The problems are generally so complex, because of various conflicting objectives, that finding "the optimal" plan is impossible. The speed that computers bring to evaluating and constructing plans has indeed made it easier for partisan groups to generate plans according to their own desires (Torricelli and Porter, 1979). Several suggestions to remedy this situation have been made. Stern (1974), for example, proposed to use a strict mathematical definition of compactness as the sum of the areas that are within a circle circumscribing a district but outside of that district. Adams (1977) suggested that redistricting be handled by an independent federal committee and that the maintenance of political boundaries be the second most important criterion to population equality.

The State of Michigan Supreme Court (1982) attempted to avoid the problems of political interests in redistricting by defining a strict set of criteria to be used in developing redistricting plans for the state legislature. 
The main emphasis of these criteria falls on the integrity of political boundaries. The rules in order of importance are:

(1) to minimize the number of county lines broken, consistent with a population deviation of less than $16.4 \%$ (91.8 to $108.2 \%$ of the mean district population);

(2) to minimize the number of townships used in breaking county lines;

(3) to minimize the number of city and township lines broken;

(4) in cities and townships with more than one representative, to achieve maximum compactness within a population deviation of $4 \%$ among districts in that municipality.

These criteria are consistent with the court's opinion in Mahan v. Howell (1973) that preserving political boundaries is a legitimate concern of the states. The criteria define the problem more clearly and make fewer partisan choices possible. In the next section, we will formulate the problem of minimizing the number of political boundaries broken as a quadratic program. In Section 2, a heuristic solution procedure for this nonconvex problem is proposed and the results for an implementation on the Michigan Senate are presented.

\section{PROBLEM FORMULATION}

Previous methods for political redistricting have not been specifically concerned with preserving political boundaries and, therefore, do not perform well with this objective. For example, in Michigan, the largest political unit that could be used in the Garfinkel and Nemhauser approach would be a township or city. There are over 1300 of these units. Garfinkel and Nemhauser describe problems with 50 units as "very difficult." The Forrest and Weaver-Hess methods do not consider political boundary preservation, and the Nagel algorithm depends strongly on the initial solution.

Our method is to use counties (or other jurisdiction units) as the building blocks of districts but to allow those units to be divided. The goal is then to find the plan with the fewest number of divided county units. A district in our model is represented as a connected directed graph of arcs $(i, j)$ where $i$ and $j$ are counties that share a common boundary. We then define decision variables:

$y_{i k}=q$, if a fraction $q$ of county $i$ is in district $k$;

$x_{i j k}=q$, if a fraction $q$ of county $i$ is in district $k$ and at least a fraction $q$ of county $j$ adjacent to $i$ is in district $k$.

The object of the model is to construct each district $k$ such that there is one less positive $x_{i j k}$ than there are positive $y_{i k}$ 's. That is, if no cycles are formed among the arcs, the graph formed by the arcs representing positive $x_{i j k}$ connecting nodes representing positive $y_{i k}$ is a tree. The tree 
structure is important because it guarantees connectedness (contiguity) (Bondy and Murty, 1976). Checking for cycles is also easier than checking for contiguity directly because a count on the number of arcs connecting a set of nodes is all that is necessary. If the number of arcs with positive $x_{i j k}$ is equal to the number of nodes with positive $y_{i k}$ adjacent to those arcs, then a cycle occurs.

The model here differs from most graphical descriptions in its allowing for fractional $y_{i k}$ and $x_{i j k}$ values. To obtain the tree structure with values of $x_{i j k}$ and $y_{i k}$ less than one, the arcs must be directed so that the value of $x_{i j k}$ is unambiguously the fraction of county $i$ in district $k$. The tree structure then forces the district to have one less node than arc, leaving one node without an outward arc. To be consistent in requiring all county nodes with positive $y_{i k}$ to have some $x_{i j k}$ positive, a self-loop, $x_{i i k}$, is allowed for the node which has no other positive outgoing arc. This node is called the root of the tree.

Parameters of the problem are:

$$
\begin{aligned}
& D=\text { the number of districts } \\
& C=\text { the number of counties } \\
& p_{i}=\text { the population of county } \mathrm{i} \\
& \bar{p}=\text { the highest population allowed in a district, and } \\
& \underline{p}=\text { the lowest allowable district population. }
\end{aligned}
$$

We formulate the problem as a quadratic program:

$$
\min \sum_{k=1}^{D} \sum_{i=1}^{C} y_{i k}\left(1-y_{i k}\right)
$$

so that

$$
\begin{gathered}
\underline{p} \leqslant \sum_{i=1}^{C} p_{i} y_{i k} \leqslant \bar{p}, \quad k=1,2, \ldots, D ; \\
\sum_{k=1}^{D} y_{i k}=1, \quad i=1,2, \ldots, C ; \\
\sum_{\substack{i=1 \\
i \neq j}}^{C} \sum_{j=1}^{C} x_{i j k}=\sum_{i=1}^{C} y_{i k}-1, \quad k=1,2, \ldots, D ; \\
\sum_{j=1}^{C} x_{i j k}=y_{i k}, \quad k=1,2, \ldots, D, i=1,2, \ldots, C ; \\
x_{i j k} \leqslant y_{i k}, \quad 1,2, \ldots, C, j=1,2, \ldots, C, k=1,2, \ldots, D ; \\
\sum_{\substack{i=1 \\
0}} x_{i i k}=1, \quad k=1,2, \ldots, \mathrm{D} ; \\
0 \leqslant x_{i j k} \leqslant 1, \quad 0 \leqslant y_{i k} \leqslant 1, \\
i=1,2, \ldots, C, j=1,2, \ldots, C, k=1,2, \ldots, D .
\end{gathered}
$$


The constraints are used to obtain feasible districts represented by the tree structure. In discussing how feasibility is obtained by the constraints, we assume that each district contains at least one entire county. We can assure that this will occur if we partition counties with populations above $\bar{p}$ into smaller "counties" with poulations below $\tilde{p}$. The implementation of Section 2 does this by eliminating counties which can contain integral numbers of districts.

Constraint (1.1) forces district populations to be within the given limits and (1.2) ensures that all counties are assigned to some district. The other constraints are used to obtain the tree structure. Constraint (1.3) forces the sum of the arc weights in a district to be one less than the number of nodes in the district. Constraint (1.4) forces every county in district $k$ to have outward pointing arc weights equal to the fraction of that county assigned to that district. Constraint (1.5) forces every county $j$ with an inward arc $x_{i j k}$ to have at least as great a fraction $y_{j k}$ assigned to district $k$ as does county $i$, and constraint (1.6) forces every district graph to have at most one self-loop.

We will show that constraints (1.3)-(1.6) lead to a contiguous district if that district's graph of positive $x_{i j k}$ valued arcs contains no cycles. First, we show that only one outward arc $x_{i j k}$ is positive if $y_{i k}$ is positive. Assume not then some node has at least two outward arcs with positive weights, $x_{i i, k}$ and $x_{i i_{2} k}$. Without loss of generality, assume $i$ has exactly two such arcs. Since we assume there are no cycles, the paths of arcs with positive weights through $i_{1}$ and $i_{2}$ end on distinct nodes $j_{1}$ and $j_{2}$ such that no arcs with positive weights point outward from $j_{1}$ and $j_{2}$ (see Fig. 1). In this case, at least one of $y_{j 1 k}$ and $y_{j 2 k}$ equals one, because $y_{j k}$ is increasing for $j$ on the path from $i$ to $j_{1}$ or $j_{2}$ by (1.5) and at least one $y_{j k}$ equals one by assumption. In this case $x_{j v_{i k}}+x_{j j_{i j k}}>1$ which violates (1.6). Hence, every county in a district has only one arc with positive weight.

Another consequence of (1.5) is that the root node has the highest fractional assignment, which by assumption is one. Therefore, constraints (1.3) and (1.4) and the fact that every node with a positive $y_{i k}$ has only

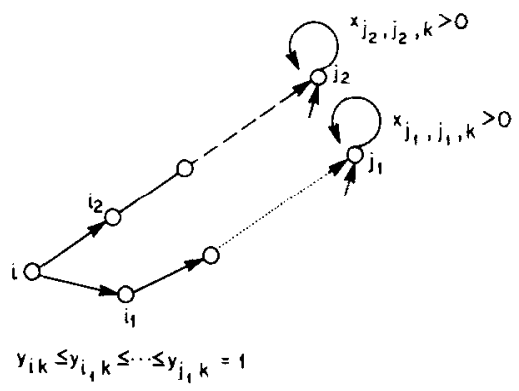

Fig. 1. Two outward ares leads to a contradiction of one self-loop. 
one positive $x_{i j k}$ force the graph of the district to have as many positive arcs as there are positive nodes minus the single root. Hence, if there are no cycles, the graph of the district is connected.

Solving (1) still requires that no cycles are present in order to obtain contiguity. In implementing the model, we can prevent cycles by adding constraints if a solution produces a graph with a cycle.

To see how this can be done, first observe that a cycle must be directed. If not, then we have two disconnected subgraphs each of which has a self-loop $\left(x_{i i k}>0\right)$. As we showed above, one of these loops has $x_{i i k}=$ 1 and (1.6) is violated (see Fig. 2).

A cycle must, therefore, be directed. Because of constraint (1.5), every $y_{j k}$ for $j$ in the cycle must be the same. By our assumption that some $y_{j k}=1$, we have all $y_{j k}=1$ on the cycle. If there are $m+1$ nodes in the cycle, we want the sum of the weights on arcs joining them to be less than or equal to $m$ to prevent a cycle. If the cycle is formed of arcs, $\left(i, j_{1}\right),\left(j_{1}, j_{2}\right), \ldots,\left(j_{m}, i\right)$, for district $k$, then the constraint for this restriction is

$$
x_{i j 1} k+\sum_{i=1}^{m-1} x_{j i j+1 k}+x_{j m i k} \leqslant m .
$$

Whenever a cycle is found, a constraint of type (1.7) is added to (1). The problem is solved again and the solution is checked for violations. The process is repeated until no violations occur.

Given a solution without cycles, the objective function is used to force the $y_{i k}$ variables to 0 or 1 , that is, to force a county to be either completely assigned to a district or not at all. It was thought that this objective would most nearly satisfy the goal of minimizing the number of divided counties while still maintaining a simple form. The quadratic term is used instead of higher powers because it allows for a global optimization procedure described briefly in Section 2 .
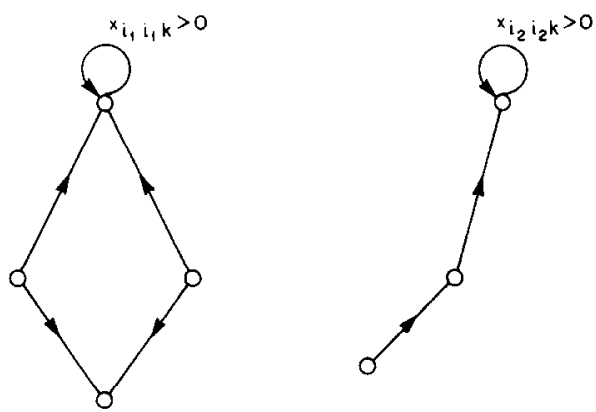

Fig. 2. An undirected cycle leads to two loops. 


\section{SOLUTION METHOD}

A full formulation of (1) would include as many as six $C D$ variables if every county was adjacent to four other counties. A large number of constraints may also be present. For the Michigan State Senate, there are, for example, 83 counties and 38 districts, yielding possibly 31,540 variables.

A heuristic to reduce the size of the program in (1) was developed using a hicrarchical clustcring procedure (see, for example, Johnson, $1967)$ and appropriate definitions of the variables. The clustering algorithm involves the following:

Step 1. Assign districts to all counties which can accommodate an integral number of districts.

Step 2. Order all adjacent pairs $(i, j)$ of the remaining counties by

$$
\delta_{i j}=\left|p^{*}-\left(p_{i}+p_{j}\right)\right|
$$

in increasing order of $\delta_{i j}$, where $p^{*}=\sum_{i=1}^{C} p_{i} / D$, the mean
population of a district.

Step 3. Proceed through the list of pairs and connect all counties into a district if

(a) $p_{i}+p_{j} \leqslant \bar{p}$, and

(b) the remaining set of counties is composed of contiguous pieces that can each support an integral number of districts.

Step 4. If no new connections were made in Step 3, stop. Else, relabel the connected counties as new counties with populations equal to the sum of the populations of the counties forming them and return to Step 2.

This algorithm results in a set of districts composed of an integral number of counties and in a set of counties which have not yet been placed into districts. The program in (1) can then be applied to the separate contiguous pieces of remaining counties. For the State of Michigan Senate, this procedure was implemented, yielding 31 districts composed of 66 whole counties.

For the remaining 17 counties and 7 districts in Michigan, a modified program (1) was solved. In this implementation, the number of districts a county could enter was restricted to reduce the number of variables. This can be done by restricting districts to general areas and observing, for example, that if a district is generally in the eastern section of the state, then no far western counties will be in it. The resulting problem in Michigan had 71 constraints and 129 variables (including slack variables).

Local optima were found using the nonlinear programming code MINOS (Murtagh and Saunders, 1977) on the University of Michigan's Amdahl 
470/V8 computer. Several problems were solved in searching for other local optima by branching on the fractional variables. After each solution, the result was checked for cycles and none were found. In searching for other local optima, if a fraction of a county was assigned to a district then that county was set at being completely in that district and the problem was solved again. In this way, many local optima were investigated.

In the plan developed for the Michigan Senate, three counties were split, including one county split among three districts. Because of the presence of two counties with populations slightly above $\bar{p}$, it could be shown that at least three counties had to be split. Therefore, the minimum number of divided counties was exactly three. Other plans, may, however, split fewer townships within the counties, but this was not considered in the optimization. This plan placed 10 districts in Wayne County. Another similar plan in which Wayne County obtained 9 districts was also developed. The latter plan also included 3 county line breaks.

The success of this procedure depends on the specific features of the formulation of (1). The clustering heuristic can be used to reduce the problem size and other analyses of the specific problem may be able to reduce the problem further. (For instance, finding two adjacent counties $i$ and $j$ such that $p \leqslant \rho_{i}+p_{j} \leqslant \bar{p}$ and, for all other $k$ adjacent to $i$ and $j, p_{i}+p_{k}>\bar{p}$ and $p_{j}+p_{k}>\bar{p}$.) The program (1) can then be solved for the remaining counties and districts by finding a local optimum and checking for cycles. If a cycle exists, then a constraint (1.7) is added and the problem is re-solved until no cycles exist.

For the Michigan Senate, after investigating several local optima, it was possible to show that the best solution found was indeed a global optimum. In general, additional constraints called Tui (1964) cuts may be added to (1) (Cabot, 1974) and a global optimum may be found by ranking the extreme point local optima (Cabot and Francis, 1970; Murty, 1968). These methods rely on the quadratic form of the objective as is present in (1). The procedures may, however, involve a large number of solutions of (1), and our experience indicates that investigating local optima by varying the initial conditions is sufficient for finding nearly optimal districting plans. The extreme point ranking approaches may still be very valuable in providing bounds on the global optimal solution value.

\section{CONCLUSION}

A formulation of a problem in redistricting legislatures was presented with the objective of minimizing the number of existing political units that do not belong to a single district. The full formulation was shown to be extremely large as the number of districts and existing polities becomes large. A heuristic method was described for reducing the size of this problem, and its results on the Michigan Senate were presented. This procedure yielded a plan with three split counties. The State Supreme 
Court, however, accepted a plan in which four counties were split among different districts.

In this case, the Supreme Court had ordered a plan to be developed after plans submitted by the two parties were considered too partisan. Their opinion led to rules (1)-(4). The court-ordered plan attempted to meet these criteria but still allowed for more county splits than were necessary. The court heard arguments against this plan, at which time, the plan generated by the procedures of Section 3 was presented. The court's opinion stated that the arguments against their plan were not sufficient to invalidate it. The reason for this action, despite the earlier statement of criteria for districting, appeared to be that the court considered itself alone to be nonpartisan and capable of generating such a plan. Upcoming elections also forced the court to act quickly and adopt the plan they had ordered. The court plan was not overtly partisan but favored the Republican party (represented by a majority of the court) relative to the former plan.

Given an early declaration of criteria to maximize the preservation of political boundaries, the creation of optimal (at least relative to Rule 1) plans should be possible. The procedure given here may be used to create these plans and avoid partisan decisions.

\section{REFERENCES}

Adams, B. (1977), "A model state reappointment process: The continuing quest for "fair and effective representation"," Harvard Journal of Legislation 14, 825-904.

Bondy, J. A., and Murty, U. S. R. (1976), Graph Theory and Applications, American Elsevier, New York.

Cabot, A. V. (1974), "Variations on a cutting plan method for solving concave minimization problems with linear constraints," Naval Research Logistics Quarterly 21, 265-279.

Cabot, A. V., and Francis, R. L. (1970), "Solving certain nonconvex quadratic minimization problems by ranking the extreme points," Operations Research 18, 82-86.

Engstrom, R. L. (1967), "The Supreme Court and equipopulous gerrymandering: A remaining obstacle in the quest for fair and effective representation," Arizona State Law Review, 277-319.

Forrest, E. (1965), “Electronic reapportionment mapping," Data Processing Magazine 7(7), $52-54$.

Garfinkel, R. S., and Nemhauser, G. I. (1970), "Optimal political districting by implicit enumeration techniques," Management Science 16(8), B495-B500.

Guida, K. J. (1982), "Deviations and justifications: Standards and remedies in challenges to reapportionment plans," The Urban Lawyer 14(1), 57-88.

Johnson, S. (1967), "Hierarchical clustering schemes," Psychometrika 32, 241-254.

Michigan Supreme Court (1982), "In re apportionment of Michigan Legislature-1982," Michigan Reports 413(3), 96-223.

Murtagh, B. A., and Saunders, M. A. (1977), MINOS User's Guide, Systems Optimization Laboratory, Stanford University, Technical Report 77-9.

Murty, K. G. (1968), "Solving the fixed-charge problem by ranking the extreme points," Operations Research 16, 268-279.

Nagel, S. S. (1965), "Simplified bipartisan computer redistricting," Stanford Law Review 17, 863-899. 
Stern, R. S. (1974), "Political gerrymandering: A statutory compactness standard as an antidote for judicial impotence," University of Chicago Law Review 41, 398-416.

Torricelli, R. G., and Porter, J. I. (1979), "Toward the 1980 census: The reapportionment of New Jersey's congressional districts," Rutgers Journal of Computers and Law 7, $135-156$.

Tui, H. (1964), "Concave programming under linear constraints," Soviet Mathematics, $1437-1440$.

Weaver, J. B., and Hess, S. W. (1963), "A procedure for nonpartisan districting: Development of computer techniques," Yale Law Journal 73, 288. 